\title{
LISA MISSION OVERVIEW
}

\author{
K. Danzmann ${ }^{1.2}$ for the LISA Study Team
}

${ }^{1}$ Institut für Atom- und Molekiilphysik, Universität Hannover, Callinstr. 38, 30167 Hannover, Germany

${ }^{2}$.Max-Planck-Institut für Quantenoptik, Hans-Kopfermann-Str. 1,85748 Garching, Germany

\begin{abstract}
More than 80 years ago, Einstein has predicted that accelerated masses will emit gravitational waves, propagating distortions of the spacetime fabric. The gravitational wave spectrum of known and expected sources covers many decades in frequency. While sources in the audio-frequency regime above $1 \mathrm{~Hz}$ are accessible to ground-based detectors, sources in the low-frequency regime can only be observed from space because of the unshieldable background of Newtonian gravitational noise. LISA is a laser-interferometric gravitational wave detector in space designed to observe gravitational wave signals from galactic as well as cosmological sources in the frequency range from $0.1 \mathrm{mHz}$ to $1 \mathrm{~Hz}$. LISA comprises a cluster of three spacecraft at the corners of an equilateral triangle of $5 \mathrm{Mio} \mathrm{km}$ size. The cluster is in an earth-like heliocentric orbit trailing the earth by 20 degrees. Each spacecraft carries lasers and free-flying proof masses and is kept on a purely inertial orbit by drag-free technology using field emission electric propulsion.
\end{abstract}

(C) 2000 COSPAR. Published by Elsevier Science Ltd.

\section{THE NATURE OF GRAVITATIONAL WAVES}

According to Special Relativity the gravitational interaction between two bodies cannot be instantaneous because the speed of light represents the limiting speed for all interactions. Already in 1805, Laplace, in his famous Traité de Mécanique Céleste stated that, if Gravitation propagates with finite speed, the force in a binary star system should not point along the line connecting the stars, and the angular momentum of the system must slowly decrease with time. Expressed in modern language, the binary star is losing energy and angular momentum by emitting gravitational waves. In 1993, Hulse and Taylor were awarded the Nobel prize in physics for the indirect proof of the existence of Gravitational Waves using exactly this kind of observation on the binary pulsar PSR 1913+16. A direct detection of gravitational waves has not been achieved up to this day [1], [2], [3].

Einstein's paper on gravitational waves was published in 1916, but it took over fourty years, before in the late 1950 s some relativity theorists, $\mathrm{H}$. Bondi in particular, proved rigorously that gravitational radiation was in fact a physically observable phenomenon, that gravitational waves carry energy and that, as a result, a system that emits gravitational waves should lose energy [4], [5].

General Relativity replaces the Newtonian picture of Gravitation by a geometric one that is very intuitive if we are willing to accept the fact that space and time do not have an independent existence but rather are in intense interaction with the physical world. Massive bodies produce "indentations" in the fabric of spacetime, 
and other bodies move in this curved spacetime taking the shortest path, much like a system of billard balls on a springy surface. In fact, the Einstein field equations relate mass (energy) and curvature in just the same way that Hooke's law relates force and spring deformation, or phrased somewhat poignantly: spacetime is an elastic medium.

If a mass distribution moves in an asymmetric way, then the spacetime indentations travel outwards as ripples in spacetime called gravitational waves. Gravitational waves are fundamentally different from the familiar electromagnetic waves. While electromagnetic waves, created by the acceleration of electric charges, propagate IN the framework of space and time, gravitational waves, created by the acceleration of masses. are waves of the spacetime fabric ITSELF.

Unlike charge, which exists in two polarities, mass always come with the same sign. This is why the lowest order asymmetry producing electro-magnetic radiation is the dipole moment of the charge distribution, whereas for gravitational waves it is a change in the quadrupole moment of the mass distribution. Hence those gravitational effects which are spherically symmetric will not give rise to gravitational radiation. A perfectly symmetric collapse of a supernova will produce no waves, a non-spherical one will emit gravitational radiation. A binary system will always radiate [6].

Gravitational waves distort spacetime, in other words they change the distances between free macroscopic bodies. A gravitational wave passing through the Solar System creates a time-varying strain in space that periodically changes the distances between all bodies in the Solar System in a direction that is perpendicular to the direction of wave propagation. These could be the distances between spacecraft and the Earth, as in the case of the space missions ULYSSES or CASSINI (attempts were and will be made to measure these distance fluctuations) or the distances between shielded proof masses inside spacecraft that are separated by a large distance, as in the case of LISA. The main problem is that the relative length change due to the passage of a gravitational wave is exceedingly small. This does not mean that gravitational waves are weak in the sense that they carry little energy. On the contrary, a supernova in a not too distant galaxy will drench every square meter here on earth with kilowatts of gravitational radiation intensity. The resulting length changes, though, are very small because spacetime is an extremely stiff elastic medium so that it takes extremely large energies to produce even minute distortions.

\section{SOURCES OF GRAVITATIONAL WAVES}

The two main categories of gravitational waves sources for LISA are the galactic binaries and the massive black holes (MBHs) expected to exist in the centres of most galaxies.

Because the masses involved in typical binary star systems are small (a few solar masses), the observation of binaries is limited to our Galaxy. Galactic sources that can be detected by LISA include a wide variety of binaries, such as pairs of close white dwarfs, pairs of neutron stars, neutron star and black hole $\left(5--20, M_{3}\right)$ binaries, pairs of contacting normal stars, normal star and white dwarf (cataclysmic) binaries, and possibly also pairs of black holes. It is likely that there are so many white dwarf binaries in our Galaxy that they cannot be resolved at frequencies below $10^{-3} \mathrm{~Hz}$, leading to a confusion-limited background. Some galactic binaries are so well studied, especially the $X$-ray binary $4 U 1820-30$, that it is one of the most reliable sources. If LISA would not detect the gravitational waves from known binaries with the intensity and polarisation predicted by General Relativity, it will shake the very foundations of gravitational physics.

The main objective of the LISA mission, however, is to learn about the formation, growth, space density and surroundings of massive black holes (MBHs). There is now compelling indirect evidence for the existence of MBHs with masses of $10^{\overrightarrow{3}}$ to $10^{8} M_{\odot}$ in the centres of most galaxies, including our own. The 
most powerful sources are the mergers of MBHs in distant galaxies, with amplitude signal-to-noise ratios of several thousand for $10^{6} M_{\odot}$ black holes. Observations of signals from these sources would test General Relativity and particularly black-hole theory to unprecedented accuracy. Not much is currently known about black holes with masses ranging from about $100 M_{\odot}$ to $10^{6} M_{\odot}$. LISA can provide unique new information throughout this mass range.

\section{THE SPECTRUM OF GRAVITATIONAL WAVES}

Astronomical observations of electromagnetic waves cover a range of 20 orders of magnitude in frequency, from ULF radio waves to high-energy gamma-rays. Almost all of these frequencies (except for visible and radio) cannot be detected from the Earth, and therefore it is necessary to place detectors optimised for a particular frequency range (e.g. radio, infrared, ultraviolet, X-ray, gamma-ray) in space.

The situation is similar for gravitational waves. The range of frequencies spanned by ground- and spacebased detectors, as shown schematically in Figure 1, is comparable to the range from high frequency radio waves up to X-rays. Ground-based detectors will never be sensitive below about $1 \mathrm{~Hz}$, because of terrestrial

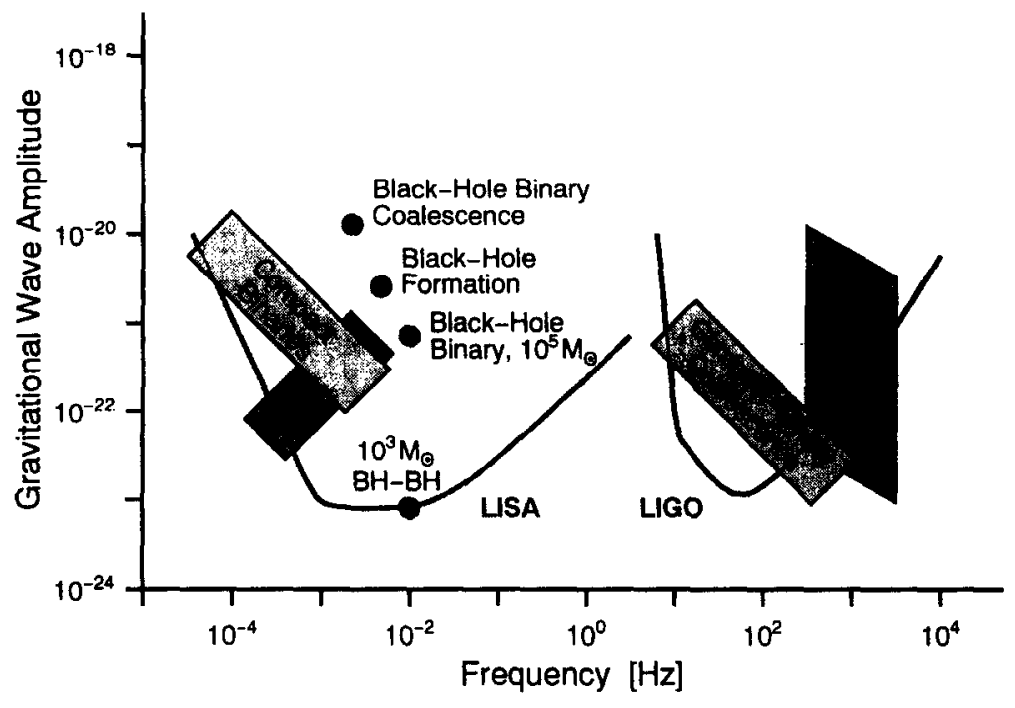

Figure 1: Comparison of frequency range of sources for ground-based and space-based gravitational wave detectors. Only a few typical sources are indicated, ranging in frequency from the $\mathrm{kHz}$ region of supernovae and final mergers of binary stars down to $\mathrm{mHz}$ events due to formation and coalescence of supermassive black holes, compact binaries and interacting white dwarf binaries. The sources shown are in two clearly separated regimes: events in the range from, say, $10 \mathrm{~Hz}$ to several $\mathrm{kHz}$ (and only these will be detectable with terrestrial antennas), and a low-frequency regime, $10^{-4}$ to $10^{-1} \mathrm{~Hz}$, accessible only with a space project. Sensitivities of LISA for periodic sources, and of (the "Advanced") LIGO for burst sources, are indicated.

gravity-gradient noise. A space-based detector is free from such noise and can be made very large, thereby opening the range from $10^{-4} \mathrm{~Hz}$ to $1 \mathrm{~Hz}$, where both the most certain and the most exciting gravitationalwave sources radiate most of their power.

The importance of low frequencies is a consequence of Newton's laws. For systems involving solar-mass objects, lower frequencies imply larger orbital radii, and the range down to $10^{-4} \mathrm{~Hz}$ includes sources with the typical dimensions of many galactic neutron star binaries, cataclysmic binaries, and some known binaries. These are the most certain sources. For highly relativistic systems, where the orbital velocities approach the speed of light, lower frequencies imply larger masses $(M \propto 1 / f)$, and the range down to $10^{-4} \mathrm{~Hz}$ reaches 
masses of $10^{7} M_{\odot}$, typical of the black holes that are believed to exist in the centres of many, if not most, galaxies. Their formation and coalescences could be seen anywhere in the Universe and are among the most exciting of possible sources. Detecting them would test the strong-field limit of gravitational theory and illuminate galaxy formation and quasar models.

For ground-based detectors, on the other hand, their higher frequency range implies that even stellar-mass systems can last only for short durations, so these detectors will mainly search for sporadic short catastrophic events (supernovae, coalescing neutron-star binaries). Normally, several detectors are required for directional information. If such events are not detected in the expected way, this will upset the astrophysical models assumed for such systems, but not necessarily contradict gravitation theory.

By contrast, if a space-based interferometer does not detect the gravitational waves from known binaries with the intensity and polarisation predicted by General Relativity, it will undermine the very foundations of gravitational physics. Furthermore, even some highly relativistic events, such as massive black hole coalescences with masses below $10^{5} M_{\odot}$, last roughly a year or longer. This allows a single space-based detector to provide directional information as it orbits the Sun during the observation.

Both ground- and space-based detectors will also search for a cosmological background of gravitational waves. Since both kinds of detectors have similar energy sensitivities, their different observing frequencies are ideally complementary: observations can supply crucial spectral information.

The space-based interferometer proposal has the full support of the ground-based detector community. Just as it is important to make observations at radio, optical, X-ray, and all other electromagnetic wavelengths, so too is it important to cover different gravitational-wave frequency ranges. Ground-based and space-based observations will therefore complement each other in an essential way.

\section{LASER INTERFEROMETERS}

Although the seeds of the idea can be found in early papers by Pirani [7] and Gertsenshtein and Pustovoit [8], it was really in the early 1970 s that the idea emerged that laser interferometers might have a better chance of detecting gravitational waves, mainly promoted by Weiss [9] and Forward [10].

A Michelson interferometer measures the phase difference between two light fields having propagated up and down two perpendicular directions, i.e. essentially the length difference between the two arms (see Figure 2). This is the quantity that would be changed by a properly oriented gravitational wave. The phase difference measured can be increased by increasing the armlength, or, equivalently, the interaction time of the light with the gravitational wave, up to an optimum for an interaction time equal to half a gravitational wave period. For a gravitational wave frequency of $100 \mathrm{~Hz}$ this corresponds to five milliseconds or an armlength of $750 \mathrm{~km}$. On the ground it is clearly impractical to build such large interferometers, but there are ways to increase the interaction time without increasing the physical armlength beyond reasonable limits. Several variants have been developed, all of them relying on storing and enhancing the laser light, or the gravitational-wave induced sidebands, or both.

The technology and techniques for such interferometers have now been under development for nearly 30 years. After pioneering work at MIT, other groups at Munich/Garching, at Glasgow, then Caltech, Paris/Orsay, Pisa, and later in Japan, also entered the scene. Their prototypes range from a few meters up to 30 , 40 , and even $100 \mathrm{~m}$.

Today, these prototype interferometers are routinely operating at a displacement noise level of the order 


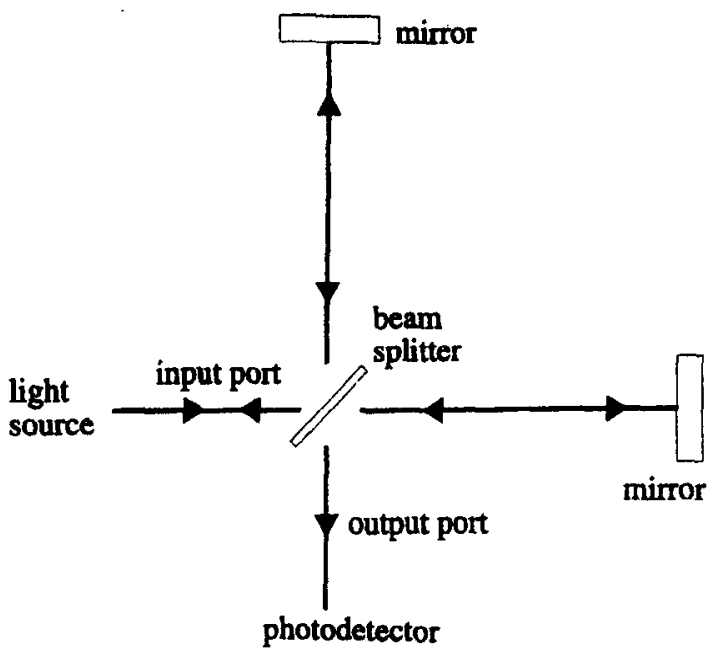

Figure 2: Schematic of a two-arm Michelson interferometer. Interference of the two returning beams on the photodetector gives a measure of their relative phase. Any variation in the mirror distances caused by a passing gravitational wave will modulate this phase signal. By having two arms fed from the same light source, the phase noise inherent to the non-ideal source is the same in each arm. In essence, the interferometry amounts to a differencing of the phases from the light returning along each arm, so the common-mode noise in the light is cancelled. This is the main reason for having two arms in a Michelson interferometer.

$10^{-19} \mathrm{~m} / \sqrt{\mathrm{Hz}}$ over a frequency range from $200 \mathrm{~Hz}$ to $1000 \mathrm{~Hz}$, corresponding to an rms gravitational-wave amplitude noise level of $h_{\mathrm{rms}} \approx 10^{-19}$.

Plans for kilometer-size interferometers have been developed for the last 15 years. All of these large-scale projects will use low-noise Nd:YAG lasers (wavelength $1.064 \mu \mathrm{m}$ ), pumped with laser diodes, just as is intended for LISA, which will greatly benefit from their efforts for achieving extreme stability and high overall efficiency.

The US project LIGO [11] for two $4 \mathrm{~km}$ interferometers, the French-Italian VIRGO [12] project for a $3 \mathrm{~km}$ interferometer, the $600 \mathrm{~m}$ long German-British GEO 600 [13] detector and the Japanese TAMA 300 [14] interferometer are scheduled to be completed by the end of this century. Observations may begin in 2000 or 2001 , although the sensitivity of the first stage detectors may be only marginally sufficient to detect gravitational waves. However, step-by-step improvements will be made, until the network finally reaches the advanced detector sensitivity sometime between 2005 and 2010. At that point, one can be confident that signals will be observed from sources such as supernovae, compact binary coalescences and pulsars, unless something is fundamentally wrong with our current estimates of their strength and distribution.

\section{THE LISA MISSION}

The LISA mission comprises three identical spacecraft located $5 \times 10^{6} \mathrm{~km}$ apart forming an equilateral triangle. LISA is basically a giant Michelson interferometer placed in space, with a third arm added to give independent information on the two gravitational wave polarizations, and for redundancy. The distance between the spacecraft - the interferometer arm length - determines the frequency range in which LISA can make observations; it was carefully chosen to allow for the observation of most of the interesting sources of gravitational radiation. The centre of the triangular formation is in the ecliptic plane, $1 \mathrm{AU}$ from the Sun and $20^{\circ}$ behind the Earth. The plane of the triangle is inclined at $60^{\circ}$ with respect to the ecliptic. These 


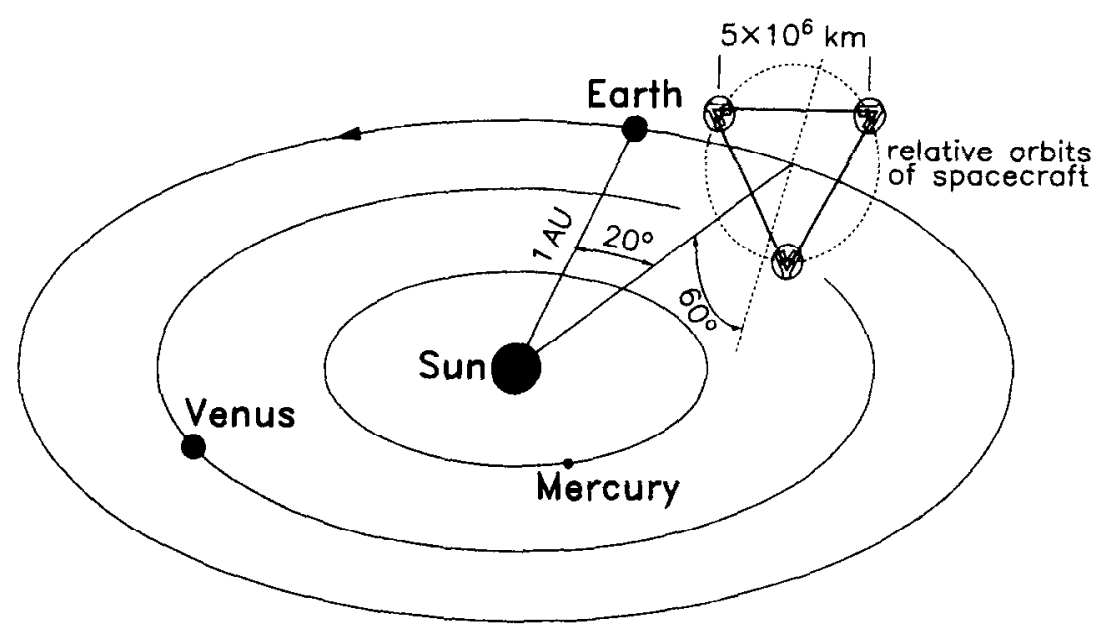

Figure 3: Three spacecraft in a triangle, with one at each vertex.

particular heliocentric orbits for the three spacecraft were chosen such that the triangular formation is maintained throughout the year with the triangle appearing to rotate about the centre of the formation once per year (Figure 3).

While LISA can be described as a big Michelson interferometer, the actual implementation in space is very different from a laser interferometer on the ground and is much more reminiscent of the technique called spacecraft tracking, but here realized with infrared laser light instead of radio waves. The laser light going out from the center spacecraft to the other corners is not directly reflected back because very little light intensity would be left over that way. Instead, in complete analogy with an RF transponder scheme, the laser on the distant spacecraft is phase-locked to the incoming light providing a return beam with full intensity again. After being transponded back from the far spacecraft to the center spacecraft, the light is superposed with the on-board laser light serving as a local oscillator in a heterodyne detection. This gives information on the length of one arm modulo the laser frequency. The other arm is treated the same way, giving information on the length of the other arm modulo the same laser frequency. The difference between these two signals will thus give the difference between the two arm lengths (i.e. the gravitational wave signal). The sum will give information on laser frequency fluctuations.

Each spacecraft contains two optical assemblies. The two assemblies on one spacecraft are each pointing towards an identical assembly on each of the other two spacecraft to form a Michelson interferometer. A $1 \mathrm{~W}$ infrared laser beam is transmitted to the corresponding remote spacecraft via a $30-\mathrm{cm}$ aperture $f / 1$ Cassegrain telescope. The same telescope is used to focus the very weak beam (a few $\mathrm{pW}$ ) coming from the distant spacecraft and to direct the light to a sensitive photodetector where it is superimposed with a fraction of the original local light. At the heart of each assembly is a vacuum enclosure containing a freeflying polished platinum-gold cube, $4 \mathrm{~cm}$ in size, referred to as the proof mass, which serves as an optical reference ("mirror") for the light beams. A passing gravitational wave will change the length of the optical path between the proof masses of one arm of the interferometer relative to the other arm. The distance fluctuations are measured to sub-Angstrom precision which, when combined with the large separation between the spacecraft, allows LISA to detect gravitational-wave strains down to a level of order $\Delta \ell / \ell=10^{-23}$ in one year of observation, with a signal-to-noise ratio of 5 .

The spacecraft mainly serve to shield the proof masses from the adverse effects due to the solar radiation pressure, and the spacecraft position does not directly enter into the measurement. It is nevertheless necessary to keep all spacecraft moderately accurately $\left(10^{-8} \mathrm{~m} / \sqrt{\mathrm{Hz}}\right.$ in the measurement band) centered on their 


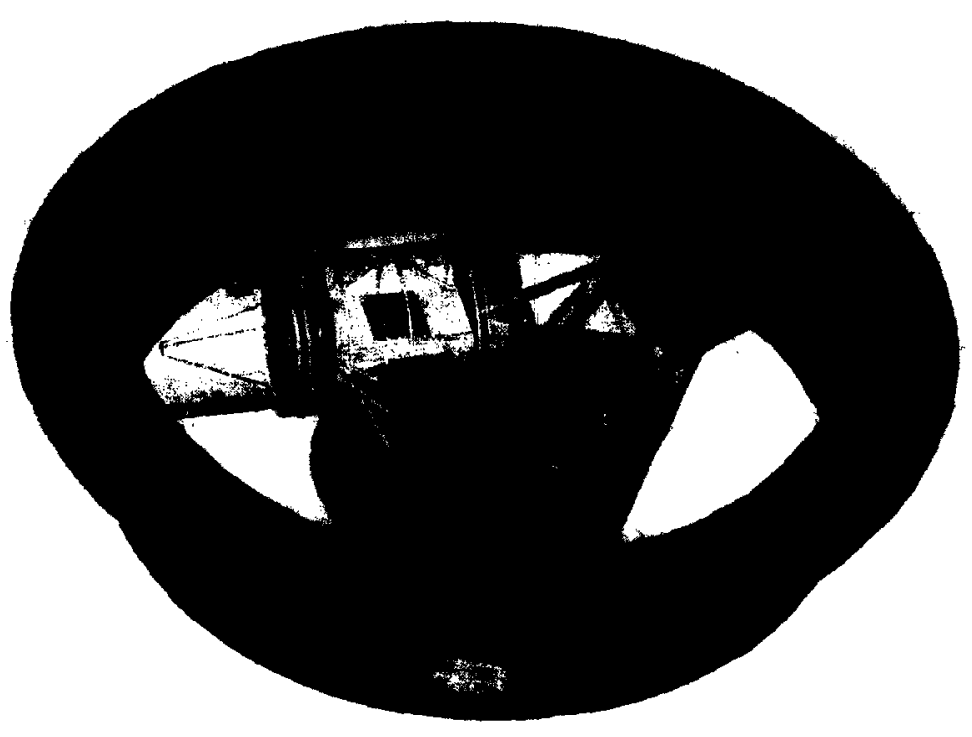

Figure 4: One of the three identical LISA spacecraft. The main structure is a ring with a diameter of $1.8 \mathrm{~m}$, and a height of $0.48 \mathrm{~m}$, made from graphite-epoxy for low thermal expansion. A lid on top of the spacecraft is removed to allow view at the $Y$-shaped thermal shield (indicated here as semitransparent) encasing the two payload arms.

respective proof masses to reduce spurious local noise forces. This is achieved by a "drag-free" control system, consisting of an accelerometer (or inertial sensor) and a system of electrical thrusters.

Capacitive sensing in three dimensions is used to measure the displacements of the proof masses relative to the spacecraft. These position signals are used in a feedback loop to command micro-Newton ion-emitting proportional thrusters to enable the spacecraft to follow its proof masses precisely. The thrusters are also used to control the attitude of the spacecraft relative to the incoming optical wavefronts, using signals derived from quadrant photodiodes. As the three-spacecraft constellation orbits the Sun in the course of one year, the observed gravitational waves are Doppler-shifted by the orbital motion. For periodic waves with sufficient signal-to-noise ratio, this allows the direction of the source to be determined (to arc minute or degree precision, depending on source strength).

Each of the three LISA spacecraft (shown in Figure 4) has a launch mass of about $400 \mathrm{~kg}$ (plus margin) including the payload, ion drive, all propellants and the spacecraft adapter. The ion drives are used for the transfer from the Earth orbit to the final position in interplanetary orbit. All three spacecraft can be launched by a single Delta II $7925 \mathrm{H}$. Each spacecraft carries a $30 \mathrm{~cm}$ steerable antenna used for transmitting the science and engineering data, stored on board for two days, at a rate of $7 \mathrm{kBps}$ in the $\mathrm{Ka}$-band to the 34-m network of the DSN. Nominal mission lifetime is two years.

LISA is envisaged as a NASA/ESA collaborative project, with NASA providing the launch vehicle, the Kaband telecommunications system on board the spacecraft, mission and science operations and about $50 \%$ of the payload, ESA providing the three spacecraft including the ion drives, and European institutes, funded nationally, providing the other $50 \%$ of the payload. The collaborative NASA/ESA LISA mission is aimed at a launch in the 2008-2010 time frame. 


\section{References}

[1] C.W. Misner, K.S. Thorne, and J.A. Wheeler, Gravitation (Freeman \& Co., San Francisco, 1973).

[2] P.R. Saulson, Fundamentals of Interferometric Gravitational Wave Detectors (World Scientific, Singapore, 1994).

[3] B.F. Schutz, A First Course in General Relativity (Cambridge University Press, Cambridge, 1985).

[4] K.S. Thorne, Gravitational Radiation, in: S.W. Hawking and W. Israel, eds., 300 Years of Gravitation (Cambridge University Press, Cambridge, 1987) 330-458.

[5] J. Weber, Phys. Rev. 117 (1960) 306.

[6] M. Bassan, Class. Quant. Grav. Supplement A 39 (1994) 11.

[7] F.A.E. Pirani, Acta Physica Polonica 15 (1956) 389.

[8] M.E. Gertsenshtein and V.I. Pustovoit, JETP 16 (1963) 433.

[9] R. Weiss, Quarterly Progress Report of RLE, MIT 105 (1971) 54.

[10] G.E. Moss, L.R. Miller, and R.L. Forward, Appl. Opt. 10 (1971) 2495.

[11] A. Abramovici et al., Science 256 (1992) 325.

[12] G. Bradaschia et al., Nucl. Instrum. and Methods A 289 (1990) 518.

[13] K. Danzmann et al., GEO600-A 300 m laser-interferometric gravitational wave antenna, Proc. $1^{\text {st }}$ Edoardo Amaldi Conference, Frascati, June 1994; and also: J. Hough et al., Proc. MG7, Stanford, July 1994.

[14] K. Tsubono and TAMA collaboration, TAMA Project in: K. Tsubono, M.-K. Fujimoto, K. Kuroda, (Eds.), Gravitational Wave Detection, Proc. TAMA Intern. Workshop, Nov. 1996, p. 183-191, Universal Academy Press (Tokyo, 1997).

[15] V.M. Kaspi, J.H. Taylor, M.F. Ryba, Astrophys. J. 428 (1994) 713. 